\title{
Root Eminence
}

National Cancer Institute

\section{Source}

National Cancer Institute. Root Eminence. NCl Thesaurus. Code C90600.

The convex bulge in the gum below a tooth caused by the underlying structure of the tooth root. 